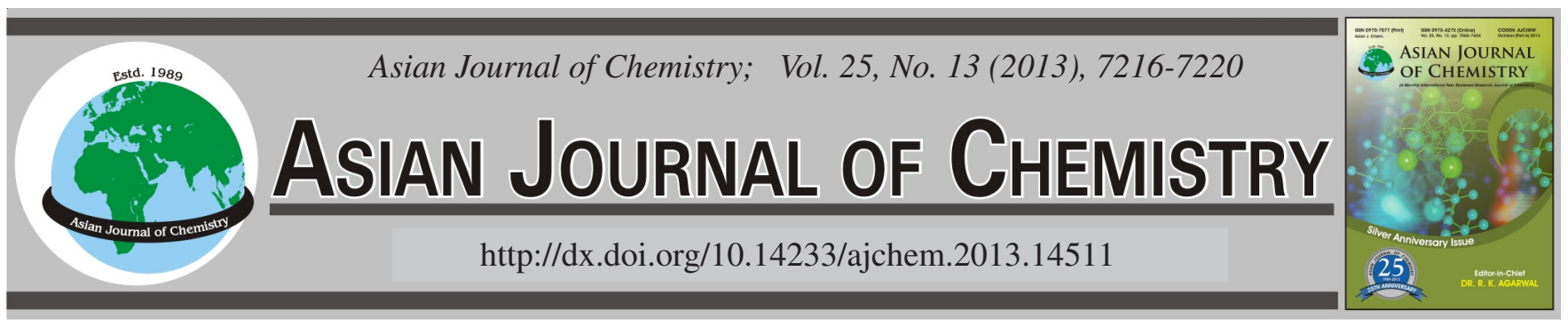

\title{
Synthesis and Characterization of the Novel NaA@Mesosilica Molecular Sieves with Core-Shell Structure
}

\author{
Shou-Gao Li, Guo-Qiang Song, Ying Meng, Hui-Shu Zhang and Fu-Xiang Li*
}

Research Institute of Special Chemicals, Taiyuan University of Technology, Taiyuan 030024, Shanxi Province, P.R. China

*Corresponding author: Fax: +86 351 6111178; Tel: +86 351 6010550-3; E-mail: L63f64x@ 163.com

\begin{abstract}
The core-shell NaA@Mesosilica molecular sieves have been synthesized with the microporous NaA zeolites as core and the mesoporous silica as a shell by using cetyltrimethylammonium bromide (CTAB) as template agent and tetraethyl orthosilicate (TEOS) as silica source in an extremely dilute liquid environment. The products are characterized by X-ray diffraction, scanning electronic microscopy, transmission electron microscopy and $\mathrm{N}_{2}$ absorption-desorption. The results show the microporous $\mathrm{NaA}$ zeolites are coated by mesoporous silica and mesoporous silica shell can grow vertically along surface of NaA zeolites. Furthermore, after calcium ion exchange, the surface area is as high as $c a .615 \mathrm{~m}^{2} / \mathrm{g}$ and the pore volume is as $c a .0 .284 \mathrm{~m}^{3} / \mathrm{g}$.
\end{abstract}

Key Words: Microporous zeolite, Mesoporous silica, Core-shell, Ion exchange.

\section{INTRODUCTION}

Since the scientists of Mobil corporation synthesized the series of M41S molecular sieves, the method using the organic template as surfactant to prepare the novel and ordered mesoporous material has become a new field ${ }^{1}$. Due to the continuous study on the structures, properties and applications of the mesoporous materials, the synthesis of the isotactic materials with various structures and morphologies have attracted considerable attentions. Nevertheless, because of the amorphous structure of the bore wall and the poor hydrothermal stability, the application of molecular sieve is greatly restricted. Microporous zeolite materials with uniform and molecularsize pores exhibit several excellent properties such as strong acidity, isotactic pore structure and good hydrothermal stability and have been widely applied in ion exchange, separation science and shape selective catalysis ${ }^{2-7}$. Whereas, the smaller surface area and aperture have limited the effective diffusion of the macromolecule, which hindered the effective transformation of the macromolecular to some extent.

It is hoped that the composite materials with not only large and homogeneous mesoporous but also microporous zeolite pore-wall structures are synthesized. For instance, Karlsson et al. ${ }^{8}$, firstly reported the preparation of MCM41/FAU composite materials and demonstrated the synthetic materials with the advantages of micro-mesoporous. Since then, numerous micro-mesoporous composite materials have been synthesized, which promoted the application of molecular sieve. However, because the smaller aperture of micropore restricted some of the molecular diffusion and material flow, the micropores were difficult to grow in the mesopores, or the mesopores in the micropores, seriously limiting the applications of the micromesoporous materials in catalytic activity ${ }^{9-16}$. There are many synthetic methods about packaging a zeolite on the surface of another molecular sieve, such as the in situ crystallization ${ }^{17}$, ex situ crystallization ${ }^{18}$, nanoclusters self-assemble ${ }^{19}$, the transformation method ${ }^{20}$ and so on. Unfortunately, the mesoporous materials synthesized by conventional process arranged irregularly in size and order degree ${ }^{21}$. As a consequence, it is necessary to invent a new way to synthesize mesoporous materials with regular surface.

In recent years, the core-shell zeolite, which is depending on the sol-gel principle and self-assembly principle, has been reported to have the regular mesoporous silica shell. The materials are synthetized using the traditional molecular sieves as the core and the mesosilica as shell. The multiphase synergies, which are provided by the molecular sieve with two different pore structures, are of great significance in the field of study. Such material can not only provide different types of catalytic center, but also be used as the carrier of numerous noble metal catalysts ${ }^{22}$. So it is likely to be used widely as the multifunctional catalytic material in the near future. For example, in the catalytic cracking reaction, the macromolecules is cracked into small molecules by the mesoporous shell firstly 
and then the small molecules continue cracking after entering into the nuclear phase and finally the desired product is obtained. Yu et al..$^{23}$, first reported a simple route to synthesize the mesoporous shell parceled by the mesoporous silica and found the as-prepared sample had a good effect in the oil catalytic cracking. They adjusted the shell thickness and mesoporous size by the control of the ratio of TEOS and C18TMS and the thermal treatment. Afterwards, Han et al. ${ }^{24}$, used CTAB as the template, silicate as the silicon source to synthesize the molecular sieve with core-shell structure in the ammonia solution. Xu et al. ${ }^{25}$, synthesized the core-shell structured molecular sieve composed by TS nuclear mesoporous silica shell with a similar approach. They used the Au metal as the carrier and applied the material to the epoxidation of propylene. Qian et al. ${ }^{26}$, synthetize the core-shell structured molecular sieve, each of which consists of the ZSM-5 nuclear mesoporous silica shell and the thickness of shell was controlled by regulating the ratio of the nuclear phase and the source of silicon. Recently, Peng et al. ${ }^{27}$, investigated the performance of coreshell structured TS@Mesosolica molecular sieves loaded with metal silver and found the molecular sieves could directly catalyze the epoxidation of propylene.

Generally, the molecular sieve with double-pore structure can not only expand the choice of the catalyst in the catalytic domain, but also separate a series of successive molecular owing to the unique core-shell structure ${ }^{28}$. Nonetheless, to our best of knowledge, little information has been done to synthetize the composite materials with the NaA molecular sieve as nuclear phase and the mesoporous silica as the shell. Therefore, we synthetize the NaA@Mesosilica molecular sieves via the calcium ion exchange method and measure the specific surface area of micropore by nitrogen adsorption technique, proving that the synthesis of mesoporous silicon layer on the NaA pore cannot cause pore blocking.

In this paper, we use the microporous $\mathrm{NaA}$ zeolites as core and the mesoporous silica as the shell to synthetize the core-shell structured NaA@Mesosilica (NaA@MS) molecular sieves with regular pore structure and larger specific surface area at an extreme dilution of the liquid environment. Meanwhile, we characterize the structure and morphology of synthetic sample and investigate the influence of synthesis conditions to its pore structure. The synthetic procedure is revealed in Scheme-I.

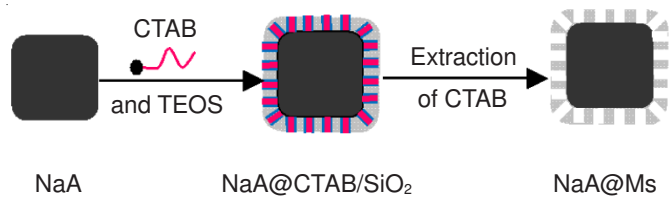

Scheme-I: Schematic diagram of the synthesis principle for NaA@MS molecular sieves

\section{EXPERIMENTAL}

$\mathrm{NaA}$ zeolites were synthesized according to a traditional hydrothermal method. For a typical procedure, a mixture containing sodium metasilicate $\left(\mathrm{Na}_{2} \mathrm{SiO}_{3} \cdot 9 \mathrm{H}_{2} \mathrm{O}\right)$, sodium aluminate $\left(\mathrm{NaAlO}_{2}\right)$ and distilled water were stirred at $30^{\circ} \mathrm{C}$ for $0.5 \mathrm{~h}$, according to the following molar composition: $\mathrm{n}\left(\mathrm{Na}_{2} \mathrm{O}\right): \mathrm{n}\left(\mathrm{Al}_{2} \mathrm{O}_{3}\right): \mathrm{n}\left(\mathrm{SiO}_{2}\right): \mathrm{n}\left(\mathrm{H}_{2} \mathrm{O}\right)=3: 1: 2: 185$ and then the reaction temperature was increased to $100{ }^{\circ} \mathrm{C}$ and maintained for $6 \mathrm{~h}$. The crystallization products were washed with distilled water for three times. Then the products were placed in the dryer that would be used as seeding. Both sodium metasilicate $\left(\mathrm{Na}_{2} \mathrm{SiO}_{3} \cdot 9 \mathrm{H}_{2} \mathrm{O}\right)$ and sodium aluminate $\left(\mathrm{NaAlO}_{2}\right)$ were purchased from Tianjin Chemical Corp (Tianjin, China). Ethanol $\left(\mathrm{C}_{2} \mathrm{H}_{5} \mathrm{OH}\right)$, cetyltrimethylammonium bromide (CTAB), concentrated ammonia solution $\left(\mathrm{NH}_{4} \mathrm{OH}, 28 \mathrm{wt} \%\right)$ and tetraethyl orthosilicate (TEOS) were purchased from Tianjin No. 3 Chemical Reagent factory (Tianjin, China). All chemicals were used as received without further purification.

Preparation of the core-shell NaA@MS molecular sieves: The core-shell NaA@MS molecular sieves were prepared by the previously reported method ${ }^{26}$. Briefly, $2.1 \mathrm{~g}$ cetyltrimethylammonium bromide (CTAB) was dissolved in a mixed solution containing $60 \mathrm{~mL}$ of distilled water and 70 $\mathrm{mL}$ of ethanol. After the solution changing clarification, 0.42 $\mathrm{g}$ obtained NaA zeolites were added to the above solution, then ultrasonic treatment for $0.5 \mathrm{~h}$ and adjusted solution $\mathrm{pH}$ to 8 with strong aqueous ammonia $\left(\mathrm{NH}_{4} \mathrm{OH}, 28\right.$ wt \%). After that, TEOS was added dropwise and stirred for $6 \mathrm{~h}$ at room temperature. In order to control the thicknesses of mesoporous silica shells, the mass ratios of TEOS/NaA were adjusted in the range of 0.2-1.6. The as-synthesised core-shell molecular sieves were washed by distilled water and ethanol. The organic templates were removed by calcination at $550{ }^{\circ} \mathrm{C}$ for $6 \mathrm{~h}$. The resulting calcined core-shell molecular sieves were named as T1, T2, T3, T4 and T5, which the mass ratios of TEOS/NaA were $0.2,0.4,0.8,1.2,1.6$, respectively.

Calcium ion exchange: The as-made core-shell sieves were added to $0.05 \mathrm{~mol} / \mathrm{L} \mathrm{CaCl}_{2}$ solution and stirred for $2 \mathrm{~h}$ at room temperature. The samples were washed by distilled water for three times and dried at $100{ }^{\circ} \mathrm{C}$ for $3 \mathrm{~h}$. Then the above step can be repeated three times.

Characterization: The XRD patterns were taken on a Japanese RigakuD/max-2500 diffractometer, using $\mathrm{CuK}_{\alpha}$ radiation $(40 \mathrm{kV}, 30 \mathrm{~mA})$. BET surface areas were measured at liquid-nitrogen temperature $\left(-196{ }^{\circ} \mathrm{C}\right)$ by American Quantachrome NOVA 2000e automated surface area and pore size analyzer. All samples were outgassed under vacuum at $300{ }^{\circ} \mathrm{C}$ for $3 \mathrm{~h}$. The total surface areas were calculated according to the BET method. Mesoporous volumes were determined by the BJH method from the desorption branch of the isotherms. Scanning electron microscopy (SEM) was obtained with German ZEISS scanning electron microscope at a voltage of $15 \mathrm{kV}$. Transmission electron mixroscopy (TEM) measurements were conducted on a Philips Tecnai G2 F20 microscope operated at $200 \mathrm{kV}$.

\section{RESULTS AND DISCUSSION}

The X-ray diffraction (XRD) patterns of the synthetic core-shell NaA@MS molecular sieves are shown in Fig. 2. Small-angle X-ray scattering (SAXS) patterns of the NaA@MS molecular sieves show one only resolved scattering peaks in the ranges $2 \theta=2.5^{\circ}$ (Fig. 1A) that can be indexed to the 100 planes of a 2-D hexagonal mesostructure with p6mm symmetry $^{20}$. That peak may be caused by the thin wall thickness ( $c a$. $25 \mathrm{~nm}$ ) of the mesoporous silica layer. The result further proves 

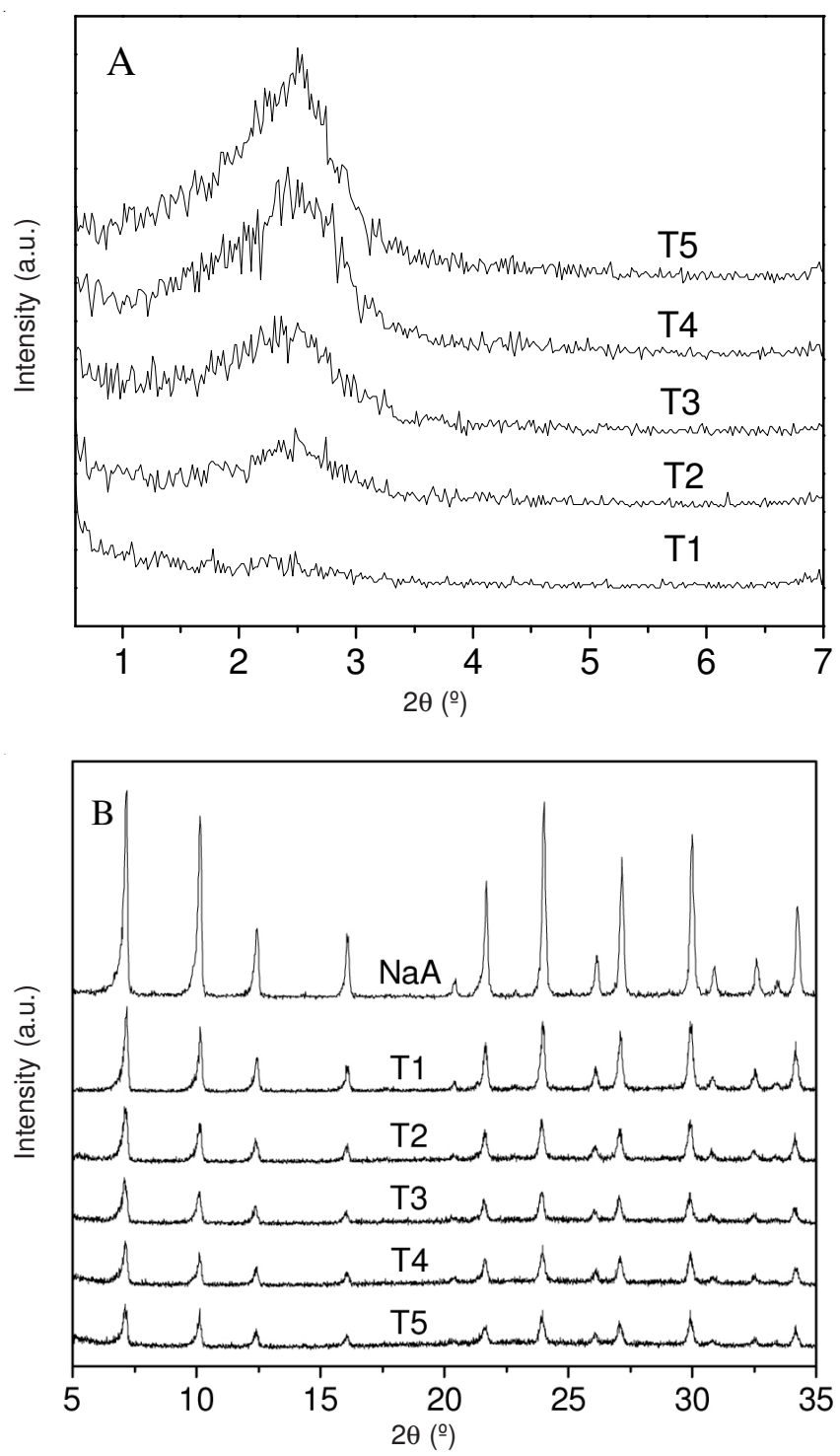

Fig. 1. (A) Small-angle XRD patterns of the core-shell NaA@MS molecular sieves B) Wide-angle XRD patterns of $\mathrm{NaA}$ zeolites and the core-shell NaA@MS molecular sieves.m(TEOS)/m(NaA): T1$0.2, \mathrm{~T} 2-0.4, \mathrm{~T} 3-0.8, \mathrm{~T} 4-1.2, \mathrm{~T} 5-1.6$

that meoprous silica can be grown on the surface of microporous NaA zeolites. With the increase of the mass of TEOS/NaA, the diffraction peaks become more and more sharp. The XRD intensity enhances with the increase of shell thickness, which is caused by the domain-size increase of the ordered mesostructure. Meanwhile, the wide-angle XRD patterns of the NaA@MS molecular sieves show the XRD pattern of the core-shell NaA@MS molecular sieves, which is similar to that of the pure $\mathrm{NaA}$ zeolites, suggesting the structure of $\mathrm{NaA}$ zeolites is retained. We also find that the diffraction peaks of NaA@MS molecular sieves have the highly crystallized NaA structures, but the intensity of the diffraction peaks of the core-shell NaA@MS molecular sieves gradually decrease with the increase of mass of TEOS/NaA, which is because of the mesoporous silica on the NaA zeolites.

The SEM image of pure NaA zeolites (Fig. 2a) shows that the as-made $\mathrm{NaA}$ zeolites (the mass ratio of TEOS/NaA = 1.2) are agglomerates of pure NaA zeolites. The high-magnification SEM image (Fig. 2b) shows that the NaA zeolites prepared have the smooth surface and obvious regular cube morphology with size of about $1 \mu \mathrm{m}$. After coating with mesoporous silica shells, the obtained NaA@MS molecular sieves (Fig. 2c) are composed of numerous agglomerates. Compared with pure $\mathrm{NaA}$ zeolites, we clearly find that there are obvious mesoporous silica shells on pure microporous $\mathrm{NaA}$
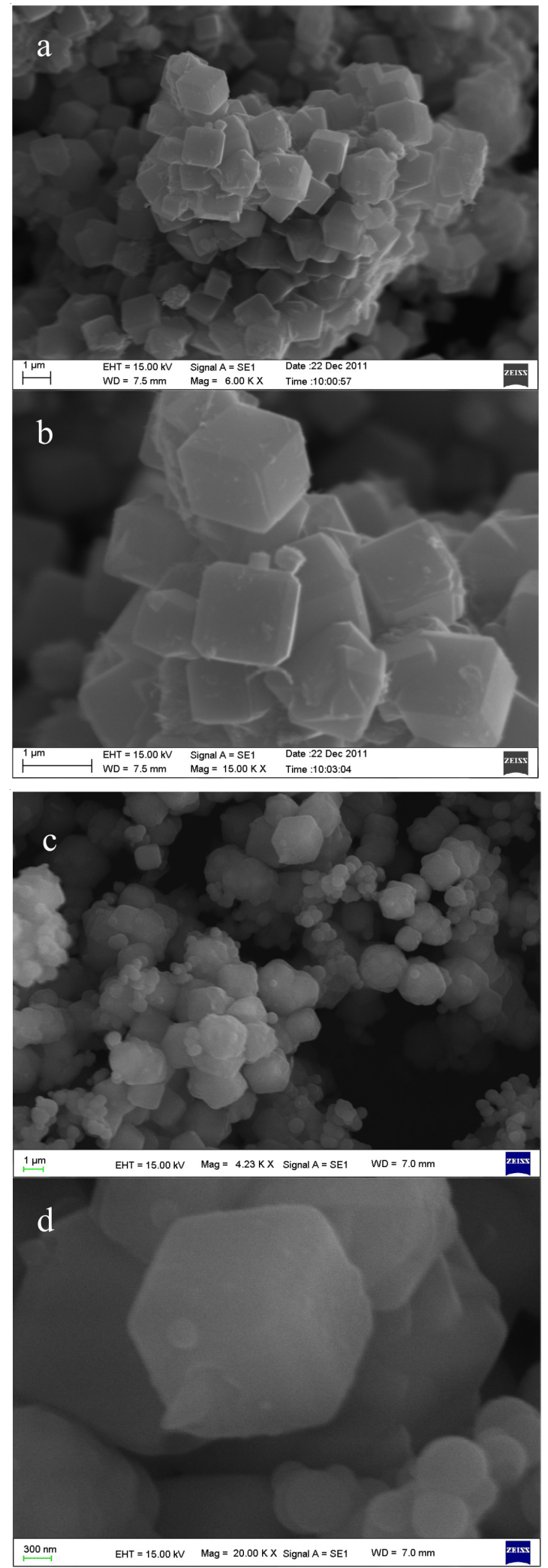

Fig. 2. SEM images of microporous NaA zeolites and NaA@MS molecular sieves with the mass ratio of TEOS $/ \mathrm{NaA}=1.2$ 
zeolites. The high-magnification SEM image (Fig. 2d) shows NaA@MS molecular sieves have a coarse surface and cube morphology, which agrees well with the morphology of $\mathrm{NaA}$ zeolites and in agreement with the XRD data. Our results show that mesoporous silica shells can grow on the microporous NaA zeolites.

Transmission electron microscopy images (Fig. 3a-d) show the as-made NaA@MS molecular sieves (the mass ratio of TEOS/NaA = 1.2) with the typical core-shell structures. Microporous NaA zeolites (Fig. 3a) are fully covered by mesoporous silica shells and their morphologies are similar to $\mathrm{NaA}$ zeolites. As the result of the thickness of mesoporous silica shells is very thin, it has little influence on shape of $\mathrm{NaA}$ zeolites. The TEM image (Fig. 3b) is the partial enlarged view of the image (Fig. 3a). We can clearly see a characteristic coreshell structure of NaA@MS molecular sieves. When the mass ratio of TEOS/NaA is 1.2, NaA single crystal (Fig. 3c) is coated uniformly with ca. $25 \mathrm{~nm}$-thick mesoporous silica shell. Highresolution TEM (HRTEM) image proves that mesoporous silica shells can grow vertically along surface of NaA zeo$\operatorname{lites}^{26}$, which is very different from the synthetic core-shell composite molecular sieves via triblock surfactant copolymer.
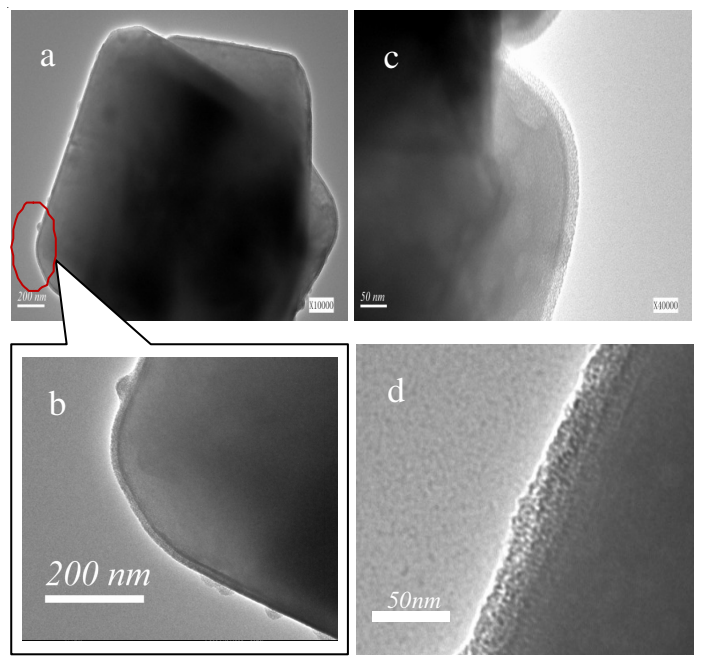

Fig. 3. TEM images of the core-shell NaA@MS molecular sieves with the mass ratio of $\mathrm{TEOS} / \mathrm{NaA}=1.2$

The nitrogen adsorption/desorption isotherms of NaA@MS molecular sieves and their corresponding pore size distributions are displayed in Fig. 4. All composite molecular sieves show type IV isotherms, suggesting uniform mesoporous structure. At higher pressure $\left(\mathrm{P} / \mathrm{P}_{0}=0.35-0.45\right)$, adsorption increases significantly, which is the mesopores in the results of capillary condensation of nitrogen. And at low relative pressure $\left(\mathrm{P} / \mathrm{P}_{0}\right)$, the adsorptions show type I curves, which suggests a typical micropore framework. These phenomena show that the synthetic samples of NaA@MS molecular sieves have double-pore structures. The adsorption isotherms of NaA@MS zeolites (Fig. 4A) reflect nitrogen capacity become strong gradually with the increase of the mass ratio of TEOS/ $\mathrm{NaA}$. That further shows that mesoporous silica shells augment the proportion of total weight. The pore size distribution of NaA zeolites and NaA@MS molecular sieves (Fig.4B) reflect mesopores centered on $2.3 \mathrm{~nm}$, which is consistent with the
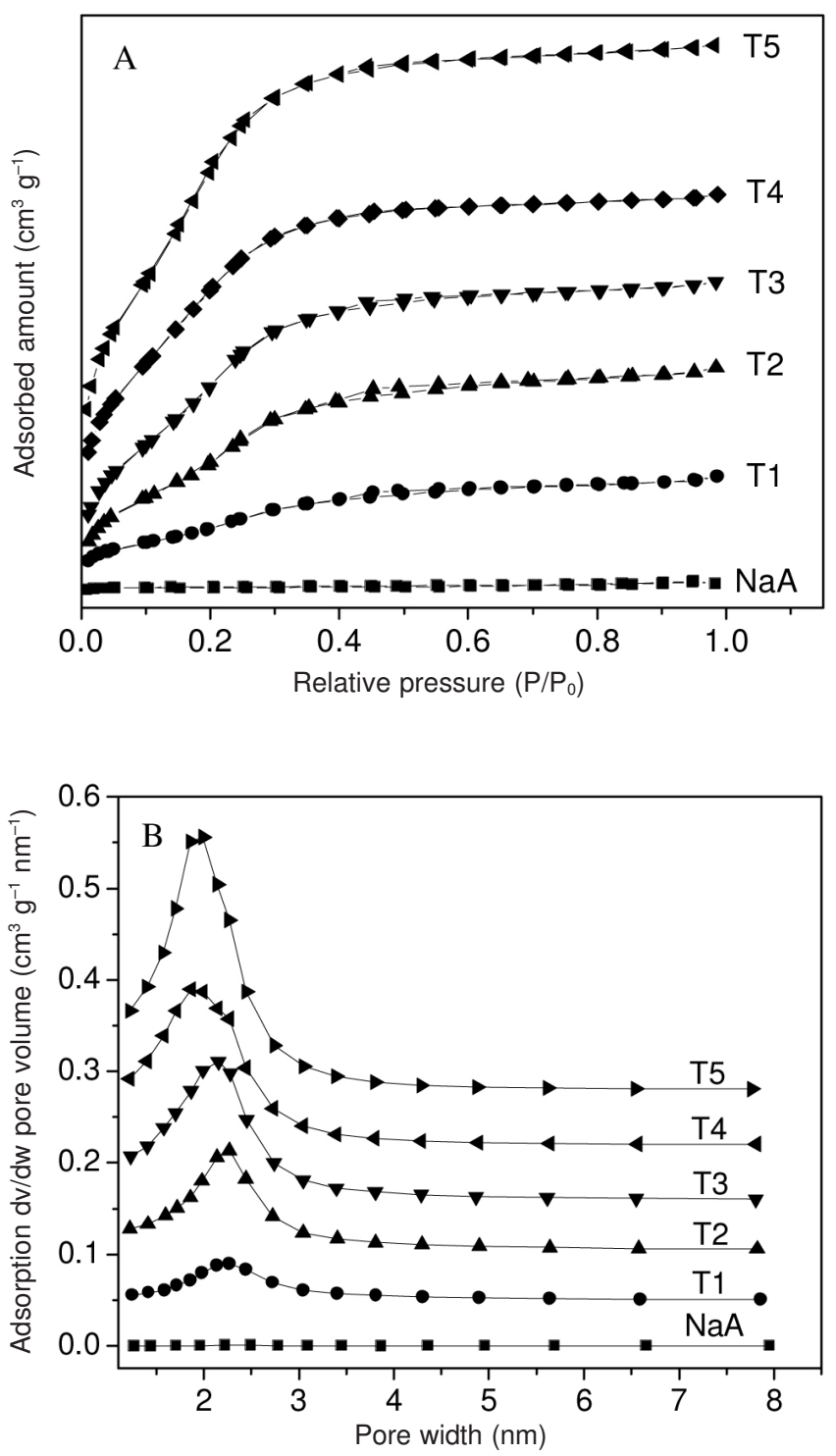

Fig. 4. $\mathrm{N}_{2}$ adsorption-desorption isotherms and mesopore size distribution of the core-shell NaA@MS molecular sieves.m(TEOS)/m(NaA): T1-0.2, T2-0.4, T3-0.8, T4-1.2, T5-1.6

pore diameter of mesoporous silica. With the increase of mass ratio of TEOS/NaA, pore size distribution becomes more and more concentrated. The results further indicate that mesoporous silica can be grown on the surface of $\mathrm{NaA}$ zeolites and the mesostructural regularity is gradually improved. Moreover, as the mass ratio of TEOS/NaA increases, physicochemical properties of NaA@MS molecular sieves change after calcium ion exchange (Table-1). From this it is revealed that microporous pore and the mesoporous shells of as-synthetic NaA@MS molecular sieves are connected to each other after using $\mathrm{Ca}^{2+}$ exchange $\mathrm{Na}^{+}$. When the mass ratio of TEOS/NaA is 1.6 , the BET surface area and total pore volume are calculated to be $615 \mathrm{~m}^{2} / \mathrm{g}$ and $0.284 \mathrm{~cm}^{3} / \mathrm{g}$. However, the BET surface and pore volume with the mass ratio of TEOS/NaA is 0.2 are calculated to be $566 \mathrm{~m}^{2} / \mathrm{g}$ and $0.058 \mathrm{~m}^{3} / \mathrm{g}$. The result is reasonable because a little mesoporous silica layers are grown on $\mathrm{NaA}$ zeolites. So it is convinced that mesoporous silica layers can be grown on microporous NaA zeolites without surface modification. 
TABLE-1

PHYSICOCHEMICAL PROPERTIES OF PRISTINE NaA AND NaA@MS AFTER CALCIUM ION EXCHANGE ${ }^{\mathrm{a}}$

\begin{tabular}{|c|c|c|c|c|c|c|}
\hline Samples & $\mathrm{S}_{\mathrm{BET}}\left(\mathrm{m}^{2} / \mathrm{g}\right)$ & $\mathrm{S}_{\text {micro }}\left(\mathrm{m}^{2} / \mathrm{g}\right)$ & $\mathrm{S}_{\mathrm{ex}}\left(\mathrm{m}^{2} / \mathrm{g}\right)$ & $\mathrm{V}_{\text {micro }}\left(\mathrm{cm}^{3} / \mathrm{g}\right)$ & $\mathrm{V}_{\mathrm{ex}}\left(\mathrm{cm}^{3} / \mathrm{g}\right)^{*}$ & $\mathrm{D}_{\text {meso }}(\mathrm{nm})^{*}$ \\
\hline $\mathrm{NaA}$ & 596 & 550 & 46 & 0.224 & - & - \\
\hline $\mathrm{T} 1$ & 566 & 436 & 130 & 0.169 & 0.058 & 2.268 \\
\hline $\mathrm{T} 2$ & 574 & 370 & 205 & 0.150 & 0.127 & 2.265 \\
\hline T3 & 587 & 263 & 323 & 0.116 & 0.178 & 2.154 \\
\hline $\mathrm{T} 4$ & 611 & 259 & 353 & 0.104 & 0.205 & 1.990 \\
\hline $\mathrm{T} 5$ & 615 & 234 & 381 & 0.103 & 0.284 & 1.998 \\
\hline
\end{tabular}

${ }^{a}$ Abbreviations: $\mathrm{S}_{\mathrm{BET}}$ : specific BET surface area; $\mathrm{S}_{\text {micro }}$ : micropore area; $\mathrm{S}_{\mathrm{ex}}:$ external surface area; $\mathrm{V}_{\text {micro }}:$ micropore volume; $\mathrm{V}_{\mathrm{ex}}:$ mesopore volume; $\mathrm{D}_{\text {meso }}$ : mesopore diameter. *Data obtained before calcium ion exchange.

\section{Conclusion}

In summary, we have synthesized successfully a novel core-shell composite molecular sieves with microporous $\mathrm{NaA}$ zeolites core and mesoporous silica shell using cationic surfactant cetyltrimethylammonium bromide (CTAB) as template agent. Mesoporous silica directly connects with microporous $\mathrm{NaA}$ zeolite due to a highly opened junction between micropore and mesopore frameworks in the core-shell structure $^{26}$. According to the text, the effect of different parameters on structural parameters of sample after calcium ion exchange has been analyzed in detail and is found that the formation of mesoporous silica is obtained when the mass ratio of TEOS/ $\mathrm{NaA}$ changes. From the present study results, core-shell structure of the molecular sieves research has just begun and its unique separation ability, effective adsorption and catalytic ability have broad application prospects.

\section{ACKNOWLEDGEMENTS}

This work was sponsored by the National Natural Science Foundation of China (No. 50972097).

\section{REFERENCES}

1. C.T. Kresge, M.E. Leonowicz, W.J. Roth, J.C. Vartuli and J.S. Beck, Nature, 358, 710 (1992).

2. D.W. Breck, Zeolite Molecular Sieves, John Wiley\& Sons: New York, p. 636 (1974).

3. J. Izumi and M. Suzuki, Adsorption, 7, 27 (2001).

4. T. Inui, M. Shibata, W. Tanakulrungsank and T. Takeguchi, Gas Sep. Purif., 6, 185 (1992).

5. D.M. Ruthven and S. Farooq, Gas Sep. Purif., 4, 141 (1990).

6. J.J. Jafar, P.M. Budd and R. Hughes, J. Membr. Sci., 199, 117 (2002).

7. F. Morón, M.P. Pina, E. Urriolabeitia, M. Menondez and J. Santamaria, Desalination, 147, 425 (2002).
8. K.R. Kloetstra, H.W. Zandbergen, J.C. Jansen and H. van Bekkum, Micropor. Mesopor. Mater., 6, 287 (1996).

9. J.C. Groen, L.A.A. Peffer, J.A. Moulijn and J. Pérez-Ramirez, Colloid. Surf. A, 241, 53 (2004).

10. J.C. Groen, T. Bach, U. Ziese, A.M. Paulaime-van Donk, K.P. de Jong, J.A. Moulijn and J. Pérez-Ramirez, J. Am. Chem. Soc., 127, 10792 (2005).

11. C.J.H. Jacobsen, C. Madsen, T.V.W. Janssens, H.J. Jakobsen and J. Skibsted, Micropor. Mesopor. Mater., 39, 393 (2000).

12. S.S. Kim, J. Shah and T.J. Pinnavaia, Chem. Mater., 15, 1664 (2003).

13. I. Schmidt, A. Boisen, E. Gustavsson, K. Stahl, S. Pehrson, S. Dahl, A. Carlsson and C.J.H. Jacobsen, Chem. Mater., 13, 4416 (2001).

14. A. Sakthivel, S. Huang, W. Chen, Z. Lan, K. Chen, T. Kim, R. Ryoo, A.S.T. Chiang and S. Liu, Chem. Mater., 16, 3168 (2004).

15. H. Wangmand and T.J. Pinnavaia, Angew. Chem. Int. Ed., 45, 7603 (2006).

16. Y. Tao, H. Kanoh, L. Abrams and K. Kaneko, Chem. Rev., 106, 896 (2006).

17. S.I. Cho, S.D. Choi, J. Kim and G. Kim, Adv. Fund. Mater., 14 (2004).

18. A.H. Janssen, A.J. Koster and K.P. de Jong, Angew. Chem. Int. Ed., 40, 1102 (2001).

19. M. Choi, H.S. Cho, R. Srivastava, Venkatesan, C. Venkatesan, D.H. Choi and R. Ryoo, Nat. Mater., 5, 718 (2006).

20. J.Y. Ying and J. García-Martínez, Mesostructured Zeolitic Materials, and Methods of Making and Using the Same, US Patent 7589041 B2 (2009).

21. Y. Tao, H. Kanoh, L. Abrams and K. Kaneko, Chem. Rev., 106, 896 (2006).

22. A. Corma, Chem. Rev., 97, 2373 (1997).

23. J.S. Yu, S.B. Yoon, Y.J. Lee and K.B. Yoon, J. Phys. Chem. B, 109, 7040 (2005).

24. Y. Han, P. Pitukmanorom, L. Zhao and J.Y. Ying, Small, 7, 326 (2011).

25. L. Xu, Y. Ren, H. Wu, Y. Liu, Z. Wang, Y. Zhang, J. Xu, H. Peng and P. Wu, J. Mater. Chem., 21, 10852 (2011).

26. X. Qian, J. Du, B. Li, M. Si, Y. Yang, Y. Hu, G. Niu, Y. Zhang, H. Xu, B. Tu, Y. Tang and D. Zhao, Chem. Sci., 2, 2006 (2011).

27. H. Peng, L. Xu, L. Zhang, J. Wang, Z. Wang, L. Chen and P. Wang, Sci. Sin. Chim., 42, 548 (2012).

28. J.M.J. Frechet, F. Svec and V. Smigol, Multimodal Chromatographic Separation Media and Process for Using Same, US Patent 5316680 (1994). 